04

\title{
Восстановление зависимости радиационных потерь плазмы токамака по измеренному поперечному распределению излучения
}

\author{
(C) В.И. Радченко, Д.Н. Ротман \\ Уральский фредеральный университет им. первого Президента России Б.Н. Ельцина, \\ 620002 Екатеринбург, Россия \\ e-mail: viradchenko7@gmail.com
}

Поступило в Редакцию 4 января 2021 г.

В окончательной редакции 4 февраля 2021 г.

Принято к публикации 5 февраля 2021 г.

Изложены теоретические основы определения плотности мощности электромагнитного излучения (радиационных потерь) в плазменном объеме, имеющем цилиндрическую симметрию, по данным для поперечного распределения мощности излучения, а также предлагаются методы решения этой задачи, которые сравниваются с известным методом Пирса.

Ключевые слова: метод Пирса, метод разностного отношения, метод отрезков полос излучения, радиационные потери.

DOI: 10.21883/JTF.2021.07.50950.268-20

\section{Введение}

Потери энергии плазмой на электромагнитное излучение (радиационные потери) в токамаках физически обусловлены тормозным излучением электронов, рекомбинационным излучением, характеристическим излучением атомов и ионов в плазме, а также циклотронным излучением электронов в магнитном поле. Диапазон энергии фотонов, излучаемых при радиационных потерях, простирается от радиочастотной до рентгеновской области (и не имеет четко устанавливаемых границ). Диапазон же энергии регистрируемых фотонов должен, по возможности, охватывать ту часть спектра, в которой измеряемые потери составляют основную часть от полных радиационных потерь. Ориентировочно этот диапазон лежит от $1 \mathrm{eV}$ до $10 \mathrm{keV}$. Поэтому для регистрации излучения применяются широкодиапазонные болометрические детекторы, область чувствительности которых соответствует указанному диапазону [1,2].

Плотность мощности электромагнитного излучения плазмы $\varepsilon(x, y, z)$, где $x, y, z$ - декартовы координаты элементарного излучающего объема, в токамаках одна из важнейших характеристик состояния плазмы в этих установках [3]. Далее $(x, y, z)$ - локальная декартова прямоугольная система координат с центром на оси плазменного шнура, ось $0 x$ которой направлена вдоль большого радиуса тороидальной камеры токамака, ось $0 y$ - параллельна главной оси симметрии тороидальной камеры.

В некоторых установках можно полагать, что радиационные потери обладают цилиндрической симметрией относительно оси плазменного объема (во всем объеме или в его части) [4]. Переходя в этом случае от декартовой $(x, y, z)$ к цилиндрической системе координат $(r, \varphi, z)$ и имея ввиду, что $\varepsilon=\operatorname{const}(\varphi)$, представим $\varepsilon$ как функцию двух переменных $\varepsilon(r, z)$. Если точка $z$ фиксирована, то пишут просто $\varepsilon(r)$. Функция $\varepsilon(r)$ рассчитывается на основе измерений поперечного (к осям $0 y$ и $0 z$ ) распределения мощности излучения $g(y)=d P(y, y+d y) / d y$, где $d P(y, y+d y)$ - мощность излучения, генерируемого по всей длине (вдоль оси $0 x$ ) хордовой полосы $[y, y+d y]$ (толщиной $(d z=1)$. Величина $d P(y, y+d y)$ определяется по результатам измерения мощности $d W(y)$ излучения, регистрируемого из указанной полосы болометрическим детектором, расположенным на продолжении хорды с координатой $y+d y / 2$ (см. ниже формулы (1), (2) и рис. 1). Экспериментальное распределение мощности излучения $d W(y) / d y$ является „профилем“ (изображением) плазменного шнура, полученным при хордовых измерениях. Распределение $g(y)$, используемое в теории (входит в состав уравнения Абеля (2)), связано с измеряемым распределением $d W(y) / d y$ известным множителем (см. формулу (2)), поэтому $g(y)$ так же трактуют как профиль плазменного шнура при хордовых измерениях.

Цель настоящей работы заключается в систематическом изложении методики определения зависимости $\varepsilon(r)$, имеющей цилиндрическую симметрию, по экспериментальным данным для функции $g(y)$, в разработ-

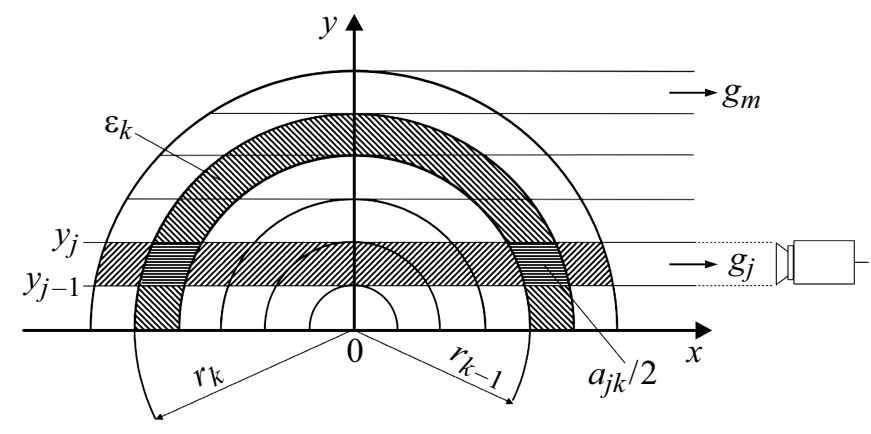

Рис. 1. Схема расчета $\varepsilon(r)$. 
ке и апробации приближений, реализующих указанную методику. Рассматриваемые вопросы представляют интерес не только с точки зрения диагностики плазмы в токамаках, но и, например, для диагностики процессов в плазменных двигателях, для диагностики цилиндрически симметричных пучков частиц в ускорительной технике.

\section{1. Определение функции $\varepsilon(r)$ по известной зависимости $g(y)$}

Выразим сначала функцию $g(y)$ через функцию $\varepsilon(r)$. Будем считать, что выполняются следующие предположения (рис. 1):

1) $\varepsilon\left(r \geq r_{m}\right)=0, r_{m}$ - наружный радиус цилиндрически симметричного плазменного объема в точке $z$ оси $0 z$;

2) на детектор (находящийся в точке $(l, y+d y / 2, z)$ ) попадает только то излучение, которое генерируется в плазменном объеме, ограниченном отрезками $\left[-x_{m}, x_{m}\right]$, $[y, y+d y],[z, z+d z]$, где $x_{m}=\sqrt{r_{m}^{2}-y^{2}} ;$ указанный плазменный объем часто называют „полосой“, $2 x_{m}$ длиной, $d y$ - шириной, $d z$ - толщиной полосы;

3) величины $d y$ и $d z$ достаточно малы, чтобы при любом фиксированном $x \in\left[-x_{m}, x_{m}\right]$ функция $\varepsilon(x, y, z)=$ $=\operatorname{const}(y, z)$ при $y \in[y, y+d y], z \in[z, z+d z]$;

$4)$ телесный угол $\Omega$, под которым виден детектор из любой точки полосы, приближенно одинаков для всех точек полосы, излучение изотропно, а плазма прозрачна для регистрируемого излучения.

В сформулированных предположениях, очевидно, что мощность излучения, падающего на детектор из указанной полосы, равна (далее $d z-$ константа задачи):

$$
\begin{aligned}
d W(y) & =2 \frac{\Omega}{4 \pi} d y d z \int_{0}^{x_{m}} \varepsilon(x, y, z) d x \\
& =\frac{\Omega}{4 \pi} d z d P(y, y+d y)=\frac{\Omega}{4 \pi} d y d z g(y) .
\end{aligned}
$$

Из предположения 3 и формулы (1) следует, что $d W(y)=\operatorname{const}(y, z)$ при $y \in[y, y+d y], z \in[z, z+d z]$.

Радиус $r$ точки в цилиндрической системе координат связан с ее координатами $x, y$ в соответствующей декартовой системе координат равенством $r=\sqrt{x^{2}+y^{2}}$. В формуле (1) $y=$ const, поэтому, дифференцируя равенство $r^{2}=x^{2}+y^{2}$, будем иметь $r(d r)=x d x$ (или $\left.d x=r d r / \sqrt{r^{2}-y^{2}}\right)$. Переходя в выражении (1) к переменной интегрирования $r$ и считая, что $y \in\left[0, r_{m}\right]$, получим соотношение между функциями $\varepsilon(r)$ и $g(y)=4 \pi d W(y) /(\Omega d z d y)$, называемое в математике уравнением Абеля:

$$
\begin{aligned}
g(y) & =\frac{d P(y, y+d y)}{d y}=\frac{4 \pi}{\Omega d z} \frac{d W(y)}{d y} \\
& =2 \int_{y}^{r_{m}} \frac{\varepsilon(r) r d r}{\sqrt{r^{2}-y^{2}}} .
\end{aligned}
$$

На практике возникает обратная задача: по известной из измерений функции $g(y)$ необходимо восстановить зависимость $\varepsilon(r)[5]$. Уравнение Абеля имеет аналитическое решение относительно $\varepsilon(r)$ [6]. Чтобы его найти, сделаем замену переменных: $t=r^{2}\left(t_{m}=r_{m}^{2}\right), s=y^{2}$. Тогда уравнение (2) примет вид

$$
g(\sqrt{s})=\int_{s}^{t_{m}} \frac{\varepsilon(\sqrt{t}) d t}{\sqrt{t-s}} .
$$

Далее вводят вспомогательную переменную $w \in[0, s]$. Переменные $w, s, t$ находятся на одной числовой оси и удовлетворяют следующим неравенствам:

$$
0 \leq w \leq s \leq t \leq t_{m}
$$

Умножим равенство (3) на $1 / \sqrt{s-w}$ и проинтегрируем по $s$ в пределах от $w$ до $t_{m}$ :

$$
\int_{w}^{t_{m}} \frac{g(\sqrt{s}) d s}{\sqrt{s-w}}=\int_{w}^{t_{m}}\left[\int_{s}^{t_{m}} \frac{\varepsilon(\sqrt{t}) d t}{\sqrt{t-s}}\right] \frac{d s}{\sqrt{s-w}} .
$$

Вычисляя по частям интеграл в левой части соотношения (5), найдем

$$
\int_{w}^{t_{m}} \frac{g(\sqrt{s}) d s}{\sqrt{s-w}}=2 g\left(\sqrt{t_{m}}\right) \sqrt{t_{m}-w} \int_{w}^{t_{m}} \frac{g_{y}^{\prime}(\sqrt{s})}{\sqrt{s}} \sqrt{s-w} d s,
$$

где $g_{y}^{\prime}(\sqrt{s})$ - производная функции $g(\sqrt{s})$ по промежуточному аргументу $y=\sqrt{s}$. Плазма заключена в объеме радиуса $r_{m}$, поэтому $g\left(\sqrt{t_{m}}\right)=g\left(r_{m}\right)=0$.

В двукратном интеграле в правой части соотношения (5) интегрирование сначала проводится (см. неравенства (4)) по переменной $t$ в пределах от $s$ до $t_{m}$, а затем по переменной $s$ в пределах от $w$ до $t_{m}$ (в итоге обе переменные принимают значения на отрезке $\left.\left[w, t_{m}\right]\right)$. Двукратный интеграл не изменится, если сначала выполнить интегрирование по переменной $s$ в пределах от $w$ до $t$, а затем по переменной $t$ в пределах от $w$ до $t_{m}$ (обе переменные интегрирования примут те же значения на отрезке $\left.\left[w, t_{m}\right]\right)$ :

$$
\begin{aligned}
& \int_{w}^{t_{m}}\left[\int_{s}^{t_{m}} \frac{\varepsilon(\sqrt{t}) d t}{\sqrt{t-s}}\right] \frac{d s}{\sqrt{s-w}} \\
& =\int_{w}^{t_{m}}\left[\int_{w}^{t} \frac{d s}{\sqrt{(t-s)(s-w)}}\right] \varepsilon(\sqrt{t}) d t=\pi \int_{w}^{t_{m}} \varepsilon(\sqrt{t}) d t .
\end{aligned}
$$

Здесь внутренний интеграл по $s$ (в квадратных скобках) легко вычисляется и равен $\pi$.

Подставим результаты (6), (7) в равенство (5), помня, что $g\left(\sqrt{t_{m}}\right)=0$ :

$$
\int_{w}^{t_{m}} \varepsilon(\sqrt{t}) d t=\frac{1}{\pi} \int_{w}^{t_{m}} \frac{g_{y}^{\prime}(\sqrt{s})}{\sqrt{s}} \sqrt{s-w} d s .
$$


Продифференцируем выражение (8) по $w$ и запишем результат в следующем виде:

$$
\varepsilon(\sqrt{w})=-\frac{1}{\pi} \int_{\sqrt{w}}^{\sqrt{t_{m}}} \frac{g_{y}^{\prime}(\sqrt{s})}{\sqrt{s-w}} d \sqrt{s} .
$$

Возвращаясь к исходным переменным и полагая $w=r^{2}$, получим искомое решение уравнения Абеля (где $\left.g_{y}^{\prime}(\sqrt{s})=g^{\prime}(y)\right)$ :

$$
\varepsilon(r)=-\frac{1}{\pi} \int_{r}^{r_{m}} \frac{g^{\prime}(y)}{\sqrt{y^{2}-r^{2}}} d y .
$$

\section{2. Метод Пирса}

При экспериментальном определении функции $g(y)$ отрезок $\left[0, r_{m}\right]$ оси $0 y$ обычно разбивается равноудаленными друг от друга точками $y_{j}(j=0,1,2, \ldots, m)$, $y_{0}=0, y_{m}=r_{m}$, задающими положение $m$ хордовых полос $\left[y_{j-1}, y_{j}\right]$, из которых ведется регистрация излучения (рис. 1) [7]. Расстояние между соседними точками обозначим через $r_{1}$. Тогда $y_{j}=j r_{1}$ и $d y=y_{j}-y_{j-1}=r_{1}(\forall j=0,1, \cdot, m)$. Заметим, что разбиение отрезка $\left[0, r_{m}\right]$ оси $0 y$ на полосы регистрации может быть иным. Полосы, задаваемые отрезком $\left[y_{j-1}, y_{j}\right]$, принято нумеровать индексом $j$ его правой границы $y_{j}$. Как следует из формул (1) и (2), мощность излучения, падающего на детектор из $j$-ой полосы, равна

$$
\Delta W_{j}=\int_{y_{j-1}}^{y_{j}} d W(y)=\frac{\Omega}{4 \pi} d z \int_{y_{j-1}}^{y_{j}} g(y) d y=\frac{\Omega}{4 \pi} d z g_{j},
$$

где величина

$$
g_{j}=\int_{y_{j-1}}^{y_{j}} g(y) d y
$$

имеет смысл мощности излучения, генерируемого в $j$-ой полосе толщиной $d z=1$. Измеряемыми величинами являются $\Delta W_{j}$ и $g_{j}$. Обратим внимание, что на рисунках излучение из $j$-ой полосы, падающее на детектор, зачастую обозначается стрелкой с символом $g_{j}$, а не $\Delta W_{j}$ (рис. 1). Очевидно, что один и тот же набор величин $g_{j}$ может быть получен для разных функций $g(y)$, т. е. найти функцию $g(y)$ однозначно из $m$ равенств (10) нельзя. Для определения $g(y)$ нужны дополнительные условия. Если на отрезке интегрирования в выражении (10) функция $g(y)$ меняется незначительно, то ее значение можно найти из равенств $g_{j} \cong g\left(y_{j}\right)\left(y_{j}-y_{j-1}\right)=g\left(y_{j}\right) r_{1}$ или $g_{j} \cong g\left(y_{j-1}\right) r_{1}$, которые означают, что $g(y)$ аппроксимирована ступенчатой зависимостью. Величины $g_{j}$ и $g\left(y_{j}\right)$ имеют близкий физический смысл, различную размерность, но их обозначают одной и той же буквой , g““.

Решение (9) уравнения Абеля, к которому приводится задача о восстановлении цилиндрически симметричной функции $\varepsilon(r)$, однозначно определяется через функцию $g(y)$. Однако (как и в случае отсутствия симметрии функции $\varepsilon(x, y, z)$, в котором задача восстановления сводится к решению интегрального уравнения Фредгольма первого рода), неоднозначность решения сохраняется изза неоднозначности определения функции $g(y)$.

Построим окружности радиуса $r_{k}=y_{k}$ $(k=1,2, \ldots, m)$ с центром в начале координат (рис. 1$)$. Примем $r_{0}=0$. Из равенства $y_{j}=j r_{1}(\forall j)$ следует, что $r_{k}=k r_{1}(\forall k=0,1, \ldots, m)$. Теперь очевиден смысл обозначений $r_{1}$ и $r_{m}$, использовавшихся выше. Кольцо, образованное соседними окружностями радиусов $r_{k-1}$ и $r_{k}$, назовем $k$-ым. Будем полагать, что ширина колец $r_{1}$ достаточно мала, чтобы в пределах любого $k$-го кольца плотность мощности излучения можно было считать постоянной и равной $\varepsilon_{k}$ (здесь $k-$ номер кольца).

Метод Пирса заключается в следующем [8]. Пусть $a_{j k}$ - площадь, образованная пересечением $k$-го кольца и $j$-ой полосы (рис. 1). Если индекс $j$ принимает одно из значений $j=1,2, \ldots, m$, то $k=j, j+1, \ldots, m$. Область, образованная указанным пересечением, является двусвязной областью (состоящей из двух зеркально симметричных участков равной площади $\left.a_{j k} / 2\right)$, в пределах которой плотность мощности излучения равна $\varepsilon_{k}$.

С учетом сказанного для величины $g_{j}$ получим формулу Пирса $(d z=1)$ :

$$
g_{j}=\sum_{k=j}^{m} a_{j k} \varepsilon_{k} \quad(j=1,2, \ldots, m) .
$$

Запишем выражение (11) в виде системы линейных алгебраических уравнений:

$$
\left\{\begin{array}{l}
g_{1}=a_{11} \varepsilon_{1}+a_{12} \varepsilon_{2}+\ldots+a_{1 m} \varepsilon_{m} \\
\ldots \ldots \ldots \ldots \ldots \ldots \ldots \ldots \ldots \ldots \ldots \ldots \ldots \ldots \ldots \ldots \\
g_{m-1}=a_{(m-1)(m-1)} \varepsilon_{m-1}+a_{(m-1) m} \varepsilon_{m} \\
g_{m}=a_{m m} \varepsilon_{m}
\end{array}\right.
$$

Все величины $g_{j}$ и $a_{j k}$ в (11), (12) известны. Для нахождения значений $\varepsilon_{k}$ возьмем сначала последнее уравнение системы (12) для $g_{m}$, из которого следует, что $\varepsilon_{m}=g_{m} / a_{m m}$. Подставляя это $\varepsilon_{m}$ в предпоследнее уравнение системы (12) для $g_{m-1}$, будем иметь $\varepsilon_{(m-1)}=\left[g_{m-1}-a_{(m-1) m} \varepsilon_{m}\right] / a_{(m-1)(m-1)}$. Запишем $j$-ое уравнение системы (12) в виде (здесь значения $\varepsilon_{k}$ для $k=j+1, \ldots, m$ уже известны):

$$
\begin{aligned}
g_{j} & =a_{j j} \varepsilon_{j}+a_{j(j+1)} \varepsilon_{j+1}+\ldots \\
& +a_{j m} \varepsilon_{m}=a_{j j} \varepsilon_{j}+\sum_{k=j+1}^{m} a_{j k} \varepsilon_{k},
\end{aligned}
$$

из которого следует, что искомое $j$-ое значение равно:

$$
\varepsilon_{j}=\frac{g_{j}-\Sigma_{k=j+1}^{m} a_{j k} \varepsilon_{k}}{a_{j j}} .
$$


Продолжая этот процесс, вычислим все $\varepsilon_{k}$. Конечно же, можно представить (11), (12) в форме матричного уравнения и найти его решение средствами алгебры матриц.

Площадь $a_{j k}$ равна $a_{j k}=s_{j k}-s_{j(k-1)}$, где $s_{j k}=$ $=S_{(j-1) k}-S_{j k}-$ площадь участка $j$-ой полосы, заключенного внутри $k$-ой окружности, здесь $S_{j k}-$ площадь кругового сегмента окружности радиуса $r_{k}$, хорда которого находится на расстоянии $y_{j}$ от центра окружности $\left(y_{j} \leq r_{k}\right.$; значит, в нашем случае $\left.j \leq k\right)$ :

$$
S_{j k}=\frac{1}{2} r_{k}^{2}\left(\theta_{j k}-\sin \theta_{j k}\right),
$$

$\theta_{j k}=2 \arccos \left(y_{j} / r_{k}\right)=2 \arccos (j / k)-$ угол дуги данного сегмента окружности. Отметим, что если $y_{j} \geq r_{k}$ $(j \geq k)$, то $S_{j k} \equiv 0$. Следовательно, $s_{j k}=0$ при $y_{j}>r_{k}$ $(j>k)$.

Формулу для $a_{j k}$ рационально использовать в следующем виде:

$$
a_{j k}=\frac{r_{1}^{2}}{2} A_{j k},
$$

где элементы $A_{j k}$ носят универсальный характер (не зависят от радиусов $\left.r_{1}, r_{m}\right)$ :

$$
\begin{aligned}
& A_{j k}=k^{2}\left[\theta_{(j-1) k}-\sin \theta_{(j-1) k}-\theta_{j k}+\sin \theta_{j k}\right]-(k-1)^{2} \\
& \times\left[\theta_{(j-1)(k-1)}-\sin \theta_{(j-1)(k-1)}-\theta_{j(k-1)}+\sin \theta_{j(k-1)}\right] .
\end{aligned}
$$

\section{3. Метод отрезков полос излучения}

Уравнение Абеля (2) для мощности $g_{j}$ излучения $j$-ой полосы имеет вид

$$
g_{j}=2 r_{1} \int_{r_{j-1}}^{r_{m}} \frac{\varepsilon(r) r d r}{\sqrt{r^{2}-r_{j-1}^{2}}} .
$$

Здесь в качестве величины $y$ из формулы (2) взята нижняя граница $j$-ой полосы $y_{j-1}$, использовано равенство $r_{j-1}=y_{j-1}$ и учтен коэффициент $r_{1}$ (см. пояснения к формуле (10)). Отрезок интегрирования в формуле (13) содержит $m-j+1$ полос, в которых координата $r \in\left[r_{k-1}, r_{k}\right], k=j, j+1, \ldots, m$. Так как в каждом из отрезков $\left[r_{k-1}, r_{k}\right]$ плотность мощности излучения неизменна и равна $\varepsilon_{k}$, равенство (13) можно записать следующим образом:

$$
g_{j}=2 r_{1} \sum_{k=j}^{m} \varepsilon_{k} \int_{r_{k-1}}^{r_{k}} \frac{r d r}{\sqrt{r^{2}-r_{j-1}^{2}}}=2 r_{1} \sum_{k=j}^{m} \varepsilon_{k} \Delta x_{j k},
$$

где после взятия интеграла введены обозначения:

$$
\begin{aligned}
& \Delta x_{j k}=x_{(j-1) k}-x_{(j-1)(k-1)} \\
& =\sqrt{r_{k}^{2}-y_{j-1}^{2}}-\sqrt{r_{k-1}^{2}-y_{j-1}^{2}} \\
& =r_{1}\left[\sqrt{k^{2}-(j-1)^{2}}-\sqrt{(k-1)^{2}-(j-1)^{2}}\right]=r_{1} l_{j k},
\end{aligned}
$$

$x_{(j-1) k}$ - координата по оси $0 x$ точки пересечения окружности радиуса $r_{k}$ с нижней границей $j$-ой полосы, пересекающей ось $0 y$ в точке $y_{j-1}$, и в формуле для $\Delta x_{j k}$ учтено, что $r_{k}=k r_{1}$, а $y_{j-1}=(j-1) r_{1}$. Величина $\Delta x_{j k}$ представляет собой длину отрезка $j$-ой полосы излучения, отсекаемого окружностями радиусов $r_{k}$ и $r_{k-1}$ от горизонтальной прямой, проходящей через точку $y_{j-1}$ и являющейся нижней границей $j$-ой полосы. В обозначении $\Delta x_{j k}$ индекс $j$ - это номер полосы. Сумма $2 \Sigma_{k=j}^{m} \Delta x_{j k}$ равна длине нижней границы $j$-ой полосы. Величины $l_{j k}$ также носят универсальный характер (не зависят от $r_{m}$ или $r_{1}$ ).

Площадь $2 r_{1} \Delta x_{j k}$ аналогична площади $a_{j k}$ в методе Пирса. Однако величины $\Delta x_{j k}$ проще в определении, нежели $a_{j k}$. Определение $\varepsilon_{k}$ выполняется точно так же, как и в методе Пирса.

\section{4. Метод разностного отношения}

Определим величину $\varepsilon_{k}$, следуя выражению (9), следующим соотношением:

$$
\varepsilon_{k}=-\frac{1}{\pi} \int_{r_{k}}^{r_{m}} \frac{g^{\prime}(y) d y}{\left(y^{2}-r_{k}^{2}\right)^{1 / 2}}
$$

считая $\varepsilon_{m}=0$ при $r_{1} \rightarrow 0$ согласно предположению 1 . Представим интеграл (15) как сумму:

$$
\varepsilon_{k}=-\frac{1}{\pi} \sum_{j=k+1}^{m} \int_{r_{j-1}}^{r_{j}} \frac{g^{\prime}(y) d y}{\left(y^{2}-r_{k}^{2}\right)^{1 / 2}}, \quad k=1,2, \ldots, m-1 .
$$

Будем полагать, что в пределах $j$-го кольца производная $g^{\prime}(y)$ постоянна и приближенно равна разностному отношению:

$$
g^{\prime}(y) \cong \frac{g\left(r_{j}\right)-g\left(r_{j-1}\right)}{r_{1}} \cong \frac{g_{j}-g_{j-1}}{r_{1}^{2}},
$$

поскольку $r_{j}-r_{j-1}=r_{1}(\forall j=1,2, \ldots, m)$. Подставим соотношение (17) в формулу (16):

$$
\varepsilon_{k} \cong \frac{1}{\pi r_{1}^{2}} \sum_{j=k+1}^{m}\left(g_{j-1}-g_{j}\right) \int_{r_{j-1}}^{r_{j}} \frac{d y}{\left(y^{2}-r_{k}^{2}\right)^{1 / 2}} .
$$

Интеграл в равенстве (18) легко вычисляется, поэтому сразу приведем результат (с учетом того, что $\left.r_{j}=j r_{1}\right)$ :

$$
\begin{aligned}
\varepsilon_{k} & \cong \frac{1}{\pi r_{1}^{2}} \sum_{j=k+1}^{m}\left(g_{j-1}-g_{j}\right) \ln \frac{j+\sqrt{j^{2}-k^{2}}}{j-1+\sqrt{(j-1)^{2}-k^{2}}} \\
& =\frac{1}{\pi r_{1}^{2}} \sum_{j=k+1}^{m}\left(g_{j-1}-g_{j}\right) L_{+} j k
\end{aligned}
$$

где логарифм $L_{j k}$ опять-таки носит универсальный характер. 
В обозначении $\varepsilon_{k}$ индекс $k$ представляет собой номер кольца, внутренний радиус которого равен $r_{k-1}$ (а в пределах $k$-го кольца значение $\varepsilon_{k}$ предполагается постоянным), поэтому в интеграле (15) величину $r_{k}$ в подынтегральной функции и на нижнем пределе интегрирования можно заменить на $r_{k-1}$. Тогда получим приближение, в котором равенства (15) и (19) предстанут в виде:

$$
\begin{aligned}
\varepsilon_{k} & =-\frac{1}{\pi} \int_{r_{k-1}}^{r_{m}} \frac{f^{\prime}(y) d y}{\left(y^{2}-r_{k-1}^{2}\right)^{1 / 2}} \cong \frac{1}{\pi r_{1}^{2}} \sum_{j=k}^{m}\left(g_{j-1}-g_{j}\right) \\
& \times \ln \frac{j+\sqrt{j^{2}-(k-1)^{2}}}{j-1+\sqrt{(j-1)^{2}-(k-1)^{2}}} .
\end{aligned}
$$

Так как величина $g_{0}$ не определена, то приближение (20) можно использовать лишь для $j \geq 2$ (а, стало быть, и для $k \geq 2$ ). Однако если вблизи начала координат (при малых $k$ ) значения $\varepsilon_{k}$ меняются несущественно, то можно положить $\varepsilon_{1}=\varepsilon_{2}$.

\section{5. Проверка методов расчета зависимости $\varepsilon_{k}$}

Для проверки той или иной методики определения зависимости $\varepsilon_{k}$ можно задать модельную зависимость $\varepsilon(r)$, с помощью этой модельной зависимости рассчитать по формуле (2) функцию $g(y)$, затем на основе $g(y)$ найти величины $g_{j}$, и, наконец, используя тестируемую методику, восстановить значения $\varepsilon_{k}$ и сравнить их с модельными. То есть надо выполнить следующую последовательность действий:

$$
\varepsilon(r) \rightarrow g(y) \rightarrow g_{j} \rightarrow \varepsilon_{k} \rightarrow \varepsilon(r)
$$

Зависимость $\varepsilon(r)$ желательно задавать в виде, который не только соответствовал бы действительности, но и позволил бы провести интегрирование при вычислении $g(y)$ и $g_{j}$ в аналитической форме. С этой целью предложим для $\varepsilon(r)$ соотношение

$$
\varepsilon(r)=\varepsilon_{0}\left(1+a \frac{r^{2}}{r_{m}^{2}}\right)\left(1-\frac{r^{2}}{r_{m}^{2}}\right),
$$

где функциональный параметр $\alpha \geq-1, \varepsilon_{0}=\varepsilon(0)$. Подставляя (21) в интеграл (2), получим

$$
g(y)=\frac{4}{3} \varepsilon_{0} r_{m}\left(1-\frac{y^{2}}{r_{m}^{2}}\right)^{3 / 2}\left[1+\alpha-\frac{4 \alpha}{5}\left(1-\frac{y^{2}}{r_{m}^{2}}\right)\right]
$$

Согласно формуле (10), мощность $g_{j}$ излучения из $j$-ой полосы с учетом (22) равна

$$
g_{j}=\int_{y_{j-1}}^{y_{j}} g(y) d y=\frac{1}{3} \varepsilon_{0} r_{m}^{2}\left[G\left(\theta_{j-1}\right)-G\left(\theta_{j}\right)\right],
$$

где

$$
\begin{aligned}
& G(\theta)=\frac{3}{2} \theta-\sin 2 \theta+\frac{1}{8} \sin 4 \theta \\
& +\alpha\left(\frac{\theta}{2}-\frac{1}{5} \sin 2 \theta-\frac{1}{40} \sin 4 \theta-\frac{1}{15} \sin ^{3} 2 \theta\right), \\
& \theta=\arccos \frac{y}{r_{m}}, \quad \theta_{j}=\arccos \frac{y_{j}}{r_{m}}=\arccos \frac{j}{m} .
\end{aligned}
$$

Теперь остается подставить найденные по формуле (23) значения $g_{j}$ в проверяемое соотношение (например, (14) или (19)) и сравнить полученные $\varepsilon_{k}$ с модельной зависимостью $\varepsilon\left(r_{k}\right)$, задаваемой выражением (21). Так как величина $\varepsilon_{k}$ постоянна внутри $k$-го кольца, то для сравнения $\varepsilon_{k}$ с модельными значениями можно предложить следующие соотношения:

$$
\varepsilon_{k} \cong \varepsilon\left(r_{k}\right), \quad \varepsilon_{k} \cong \varepsilon\left(r_{k-1}\right), \quad \varepsilon_{k} \cong\left[\varepsilon\left(r_{k-1}\right)+\varepsilon\left(r_{k}\right)\right] / 2 .
$$

Отметим, что с этой неоднозначностью связано появление двух формул (19) и (20). В настоящей работе использовалось первое из предыдущих соотношений сравнения. Результаты сравнения величин $\varepsilon_{k}$, рассчитанных методом Пирса и по формулам (14), (19), с модельной зависимостью (21) для $\alpha=3$ и $m=32$, показаны на рис. 2 (зависимости $\varepsilon_{k}$ нормированы на единицу при $k=0)$. Выбор числа $\mathrm{m}=32$ обусловлен широким применением для измерения радиационных потерь линеек (сборок) из 32 полупроводниковых болометрических детекторов [9]. В связи с этим заметим, что с ростом числа $m$ при прочих равных условиях должна, вообе говоря, улучшаться точность восстановления $\varepsilon(r)$. Но с ростом $m$ при фиксированном радиусе $r_{m}$ уменьшается ширина полосы (равная $r_{1}=r_{m} / m$ ), что приводит к увеличению относительного вклада шумов в регистрируемый из полосы сигнал и ухудшению точности восстановления $\varepsilon(r)$. В итоге компромисса между этими факторами рекомендуется [5] брать значения $m$ от 20 до 40.

В диапазоне изменения параметра $\alpha \in[-1,8]$ все три метода уверенно описывают модельную зависимость. При дальнейшем увеличении параметра $\alpha$ формула (14) дает все более и более высокие, а формула (19) - все более низкие значения $\varepsilon_{k}$ по сравнению с модельными. Десятипроцентное отличие величины $\varepsilon_{k}$ от модельных значений (в области максимума) с ростом $\alpha$ наступает: для формулы (14) при $\alpha \approx 10$, для формулы (19) при $\alpha \approx 16$, для метода Пирса при $\alpha \approx 300$.

Аналогичные расчеты были выполнены еще для двух модельных зависимостей:

$$
\varepsilon(r)=\varepsilon_{0} e^{\alpha \frac{r^{2}}{r_{m}^{2}}}\left(1-\frac{r^{2}}{r_{m}^{2}}\right)
$$

и

$$
\varepsilon(r)=\varepsilon_{0}\left(1+\alpha \frac{r}{r_{m}}\right)\left(1-\frac{r}{r_{m}}\right)
$$




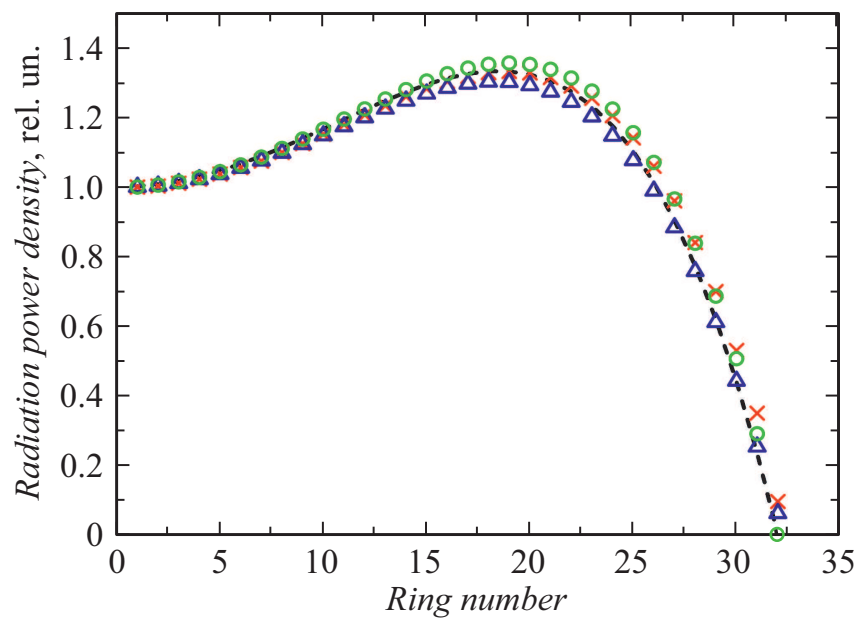

Рис. 2. Результаты расчета $\varepsilon(r)$ для $a=3:---$ модельные значения; $x-$ значения, рассчитанные методом Пирса; $\Delta-$ значения, рассчитанные методом отрезков полос излучения; о - значения, рассчитанные методом разностного отношения.

и подтвердили результаты, полученные на основе модельной зависимости (21). Это связано с тем, что различие между кривыми $\varepsilon(r)$, задаваемыми указанными зависимостями, можно сделать достаточно малым за счет выбора их параметров $\varepsilon_{0}$ и $\alpha$. Однако формула (21) предпочтительнее, поскольку дает более простые и аналитически точные выражения распределений $g(y)$ и $g$.

В настоящей работе мы не приводим результаты детального сравнения методов для различных модельных зависимостей $\varepsilon(r)$ и чисел $m$. Дело в том, что регистрируемое поперечное излучение плазмы всегда содержит шумы, вызванные колебаниями параметров плазмы, наводками в измерительной аппаратуре и т.п. Колебания параметров в некоторой точке плазменного объема приводят к искажению величины радиационных потерь в этой точке. Пусть $\varepsilon_{1}(r ; p, q), \varepsilon_{2}(r ; v, w)$ - двухпараметрические модельные зависимости радиационных потерь $\left(p, q, v, w\right.$ - их параметры), а $g_{1}(y ; p, q), g_{2}(y ; v, w)-$ соответствующие поперечные распределения излучения плазмы. Математические формулы, которыми представлены модельные зависимости, различны [5], но они предназначены для описания реальной физической зависимости $\varepsilon(r)$, поэтому подбирались так, чтобы за счет выбора параметров можно было обеспечить выполнение приближенных равенств $\varepsilon(r) \cong \varepsilon_{1}(r ; p, q) \cong \varepsilon_{2}(r ; v, w)$, а значит, при этих параметрах будет выполняться и равенство $g_{1}(y ; p, q) \cong g_{2}(y ; v, w)$. Если $\bar{N}(y)-$ математическое ожидание плотности мощности шумов в точке у поперечного распределения излучения плазмы, то в экспериментах обычно выполняется неравенство $\bar{N}(y)>\left|g_{1}(y ; p, q)-g_{2}(y ; v, w)\right|$. Таким образом, наличие шумов в зависимости $g(y)$ влияет на точность восстановления функции $\varepsilon(r)$ более значимо, чем различие в используемых модельных зависимостях (и в значениях $m$ ), что отмечалось в работе [5].

\section{Заключение}

Метод Пирса основан на анализе общей экспериментальной методики определения радиационных потерь, не используя уравнение Абеля или его решение непосредственно. Метод отрезков полос излучения построен на преобразовании уравнения Абеля (2) к виду, позволяющему найти функцию радиационных потерь. Метод разностного отношения базируется на формуле (9), дающей решение уравнения Абеля.

По результатам вычислений радиационных потерь $\varepsilon(r)$ при отсутствии шумов изложенными выше методами была показана принципиальная пригодность метода отрезков полос излучения и метода разностного отношения для восстановления зависимости $\varepsilon(r)$, а также установлено, что метод Пирса при фиксированном значении $\mathrm{m}$ является наиболее точным из рассмотренных. Однако проводить качественное сравнение методов, как следует из предыдущего разд. 5, надо по зашумленному распределению $g(y)$, предполагая, что различия в принципах построения методов позволят одному из них более точно восстанавливать зависимость $\varepsilon(r)$ при наличии шумов в составе $g(y)$.

Наши предварительные расчеты по влиянию шумов, искажающих распределение $g(y)$, на точность восстановления зависимости $\varepsilon(r)$ тремя рассмотренными методами (при использовании процедур сглаживания) показали, что с ростом уровня зашумленности $g(y)$ точность восстановления $\varepsilon(r)$ любым из трех методов резко падает (это совпадает с данными из обзора [5] для различных модельных распределений $g(y))$. Рис. 3 иллюстрирует результаты восстановления величин $\varepsilon_{k}$ данными методами по одному и тому же зашумленному на уровне $20 \%$ распределению $g_{j}$.

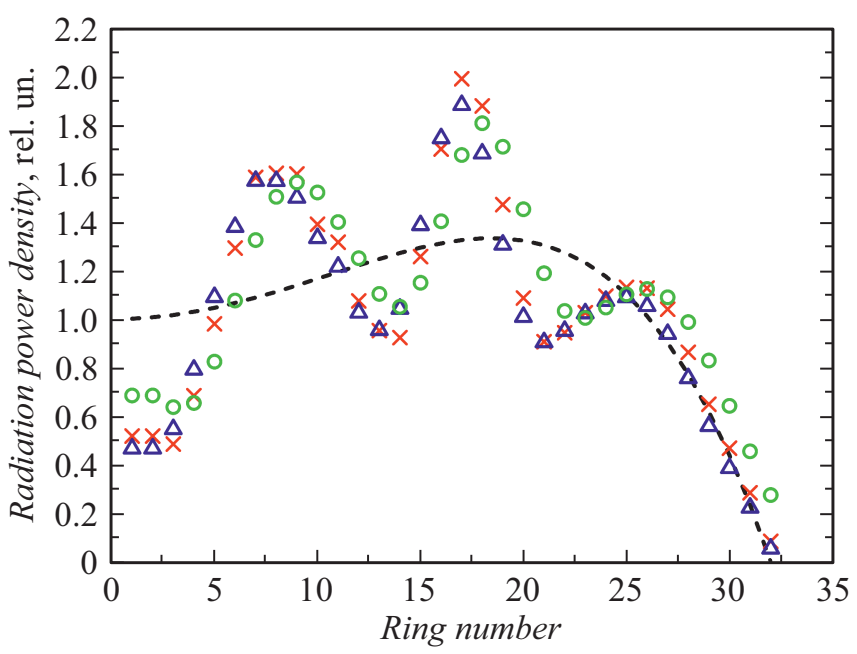

Рис. 3. Результаты восстановления зависимости $\varepsilon_{k}$ методом Пирса $(\times)$, методом отрезков полос излучения $(\triangle)$ и методом разностного отношения (о) для модельной зависимости $(21) \mathrm{c}$ параметром $a=3$ при уровне зашумленности распределения $g_{j}$, равном $20 \%$. 
Величины $\varepsilon_{k}$, восстановленные разными методами, графически ведут себя идентичным образом. Положение пиков и впадин на восстановленной зависимости $\varepsilon_{k}$ соответствует положению пиков и впадин на зашумленной зависимости $g_{j}$. Отклонение $\varepsilon_{k}$ от модельных значений в точках пика или впадины обычно составляет 80-100\% при аналогичном отклонении величин $g_{j}$ в соответствующих точках на 20-25\%. Анализ погрешности восстановления величин $\varepsilon_{k}$ показал, что в диапазоне уровня зашумленности от 10 до $35 \%$ (для $\alpha \in[0,10], m=32$ ) метод разностного отношения является более точным, чем метод Пирса и метод отрезков полос излучения (на зависимости $\varepsilon_{k}$ это систематически проявляется при значениях $k \leq 5)$. Однако преимущество метода разностного отношения невелико (10-15\% при уровне зашумленности 20-35\%).

Вопрос о восстановлении зависимости $\varepsilon(r)$ по зашумленным результатам измерения $g(y)$ является принципиально важной темой отдельных исследований и требует своего решения. Вновь разрабатываемые и существующие методы восстановления зависимости $\varepsilon(r)$ должны сравниваться между собой по точности восстановления $\varepsilon(r)$ (при заданном уровне зашумленности $g(y)$ ) на основе некоторого общего критерия сравнения.

\section{Конфликт интересов}

Авторы заявляют, что у них нет конфликта интересов.

\section{Список литературы}

[1] А.Д. Ибляминова, Г.Ф. Авдеева, П.Н. Аруев, Н.Н. Бахарев, В.К. Гусев, В.В. Забродский, Г.С. Курскиев, В.Б. Минаев, И.В. Мирошников, М.И. Патров, Ю.В. Петров, Н.В. Сахаров, С.Ю. Толстяков, П.Б. Щеголев. Физика плазмы, 42 (10), 873 (2016). DOI: 10.7868/S0367292116100048

[A.D. Iblyaminova, G.F. Avdeeva, P.N. Aruev, N.N. Bakharev, V.K. Gusev, V.V. Zabrodsky, G.S. Kurskiev, V.B. Minaev, I.V. Miroshnikov, M.I. Patrov, Y.V. Petrov, N.V. Sakharov, S.Y. Tolstyakov, P.B. Shchegolev. Plasma Phys. Reports, 42 (10), 909 (2016). DOI: 10.1134/S1063780X16100044]

[2] V.V. Zabrodsky, P.N. Aruev, V.L. Sukhanov, N.V. Zabrodskaya, B.J. Ber, D.Yu. Kasantsev, A.G. Alekseyev. In: Proceedings of the 9th International Symposium on Measurement Technology and Intelligent Instruments (D.S. Rozhdestvensky Optical Society, Saint-Petersburg, 2009), p. 3.

[3] А.Д. Ибляминова, С.Ю. Толстяков, Г.С. Курскиев, А.А. Березуцкий, Ю.В. Петров, В.В. Забродский. Научнотехнические ведомости СПбГПУ. Физико-математические науки, 158 (4), 70 (2012).

[4] Л.А. Арцимович. Избранные труды. Атомная физика и бизика плазмы (Наука, М., 1978)

[5] Э.И. Кузнецов, Д.А. Щеглов. Методы диагностики высокотемпературной плазмы (Атомиздат, М., 1974)

[6] А.Д. Полянин, А.В. Манжиров. Справочник по интегральным уравнения (Физматлит, М., 2003)

[7] A.S. Prokhorov, A.G. Alekseyev, A.M. Belov, V.B. Lazarev, S.V. Mirnov. Plasma Phys. Reports, 30 (2), 137 (2004). DOI: $10.1134 / 1.1648938$
[8] У.Д. Пирс. В сб.: Получение и исследование высокотемпературной плазмы, под ред. В.А. Фабриканта. (ИИЛ, М., 1962), c. 221.

[9] R. Korde, J. Cable, L. Canfield. IEEE Transactions on Nuclear Sci., 40 (6), 1655 (1993). DOI: 10.1109/23.273555 